\title{
Structure of a Self-Assembled Three-Dimensional DNA Crystal Framework for the Precise Organization of Biomaterials
}

\author{
Chad R. Simmons ${ }^{1,2}$, Tara MacCulloch ${ }^{1,2}$, Fei Zhang ${ }^{1,2}$, Yan Liu $^{1,2}$, Nicholas Stephanopoulos ${ }^{1,2^{*}} \&$ \\ Hao Yan ${ }^{1,2^{*}}$
}

${ }^{1}$ Center for Molecular Design and Biomimetics \& ${ }^{2}$ Department of Molecular Sciences, Arizona State University, Tempe, Arizona 85287, United States

Email Contact: chad.simmons@asu.edu

The foundational goal of the field of structural DNA nanotechnology was to assemble designed architectures facilitated by designed crystal lattices made entirely of nucleic acid structures that could precisely organize species untenable for crystallization and resulting structural determination via X-ray crystallography. ${ }^{1}$ DNA is an ideal polymer for the construction of higher order three-dimensional structures of increasing complexity due to its distinctive ability to associate via canonical Watson-Crick base pairing with highly addressable nucleotides on each array. Crystal assembly in the third dimension is facilitated via an immobile Holliday junction, a branched nucleic acid motif inspired by the structure formed during homologous recombination. Using these features, the structures of only a handful of these scaffolds have been determined. ${ }^{2-}$ ${ }^{5}$ We have used these previously determined crystal motifs to inspire the newly designed crystal lattice described here. The crystal system employs a repeating sequence that mediates the assembly of a 3D scaffold designed and dictated, via a series of Holliday junctions working in concert with specified "sticky ends" that cohere to form the array, sequence specifically. The details determined from this motif paved the way for the new high resolution (2.7 $\AA$ ) structure shown here (Fig. 1) which contained unique crystal symmetry and structural properties with atomic detail not previously achieved in any other self-assembled DNA crystal system. The crystalline scaffold was designed to yield a prescribed rhombohedral (R3) lattice that was packed in a solvent excluded environment to yield the unprecedented resolution of the structure allowing for the discrete placement of guest molecules with atomic precision within the scaffold. This system will facilitate the construction of improved three-dimensional lattices, which could in turn be used as scaffolds for immobilizing and solving the structure of molecular guests, or as catalytic materials, and provide significant insight into the mechanism of DNA self-assembly, providing the invaluable ability to design novel DNA-templated functional materials.

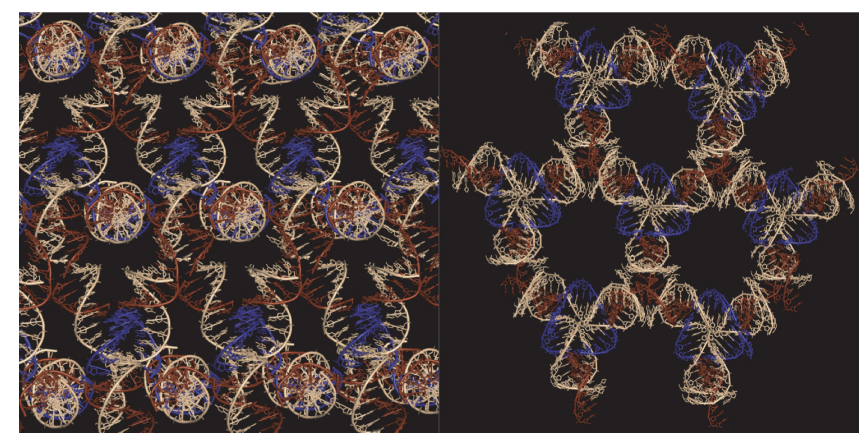

Figure 1. The self-assembled rhombohedral DNA crystal lattice.

References

[1] Seeman, N. C. (1982) J. Theor. Biol. 99: 237-247.

[2] Zheng, J. et al. (2009) Nature. 461(7260): 74-77.

[3] Simmons, C. R. et al. (2016) J. Am. Chem. Soc. 138(31): 10047-54.

[4] Simmons, C. R. et al (2017) J. Am. Chem. Soc. 139(32): 11254-60.

[5] Zhang, F. et al (2018) Angew. Chem. 57(38): 12504-12507. 